\title{
Prediction of distant metastasis of spinal and pelvic osteosarcoma by constructing and validating nomogram:a retrospective study based on SEER database
}

\author{
Yu Lin \\ Union Hospital affiliated to Fujian Medical University \\ Zhenyu Wang \\ Union Hospital affiliated to Fujian Medical University \\ Gang Chen \\ Union Hospital affiliated to Fujian Medical University \\ Wenge Liu ( $\nabla$ wengeunion@fjmu.edu.cn ) \\ Union Hospital affiliated to Fujian Medical University
}

\section{Research Article}

Keywords: Spinal and pelvic osteosarcoma, Distant metastasis, SEER database, Nomogram

Posted Date: January 13th, 2022

DOI: https://doi.org/10.21203/rs.3.rs-1235263/v1

License: (c) (i) This work is licensed under a Creative Commons Attribution 4.0 International License.

Read Full License 


\section{Abstract}

Background:Spinal and pelvic osteosarcoma is a rare type of all osteosarcomas,and distant metastasis is an important factor for poor prognosis of this disease. There are no similar studies on prediction of distant metastasis of spinal and pelvic osteosarcoma. We aim to construct and validate a nomogram to predict the risk of distant metastasis of spinal and pelvic osteosarcoma.

Methods:We collected the data on patients with spinal and pelvic osteosarcoma from the Surveillance, Epidemiology, and End Results(SEER) database retrospectively. The Kaplan-Meier curve was used to compare differences in survival time between patients with metastasis and non-metastasis. Total patients were randomly divided into training cohort and validation cohort. The risk factor of distant metastasis were identified via the least absolute shrinkage and selection operator(LASSO) regression and multivariate logistic analysis. The nomogram we constructed were validated internally and externally by C-index, calibration curves, receiver operating characteristic(ROC) curve and Decision curve analysis (DCA).

Results:The Kaplan-Meier curve showed that the survival time of non-metastatic patients was longer than that of metastatic patients $(P \otimes 0.001)$. All patients $(n=358)$ were divided into training cohort $(n=269)$ and validation cohort $(n=89)$. The LASSO regression selected five meaningful variables in the training cohort. The multivariate logistic regression analysis demonstrated that surgery (yes, $O R=0.175,95 \% \mathrm{Cl}=0.095$ $0.321, p=0.000$ ) was the independent risk factors for distant metastasis of patients with spinal and pelvic osteosarcoma. The C-index and calibration curves showed the good agreement between the predicted results and the actual results. The area under the receiver operating characteristic curve(AUC) values were $0.748(95 \% \mathrm{Cl}=0.687-0.817)$ and $0.758(95 \% \mathrm{Cl}=0.631-0.868)$ in the training and validation cohorts respectively. The DCA showed that the nomogram has a good clinical usefulness and net benefit.

Conclusion:No surgery is the independent risk factor of distant metastasis of spinal and pelvic osteosarcoma. The nomogram we constructed to predict the probability of distant metastasis of patients with spinal and pelvic osteosarcoma is reliable and effective by internal and external verification.

\section{Background}

Osteosarcoma is the most common primary malignant bone tumor,it was reported that the annual prevalence of osteosarcoma ranges $0.2-3$ cases per 100,000 people[1]. However,spinal and pelvic osteosarcoma is more rare compared with osteosarcoma in other sites.It was reported that spinal osteosarcoma accounts for $3 \%$ of all osteosarcomas, while pelvic osteosarcoma accounts for less than $10 \%$ of all osteosarcomas[2, 3].There are some studies on prediction of distant metastasis of osteosarcoma in all sites[4, 5].Nonetheless,there was no similar studies on prediction of distant metastasis of spinal and pelvic osteosarcoma because of low incidence.

Spinal and pelvic osteosarcoma has unique clinical characteristics compared with osteosarcoma in external location.Osteosarcoma of this type has a worse prognosis and higher probability to distant 
metastasize.Jiang $\mathrm{J}$ et al[6] indicated that the survival of spinal and pelvic osteosarcoma is worse than that of head and limb.A study based on SEER indicated that osteosarcoma in the axial bone is an independent prognosticator for overall survival and cancer-specific survival[7].Zhang Chao et al[5] indicated that primary tumor in axial location was significantly correlated with lung metastasis among high-grade ostoesarcoma patients. The present studies showed that distant metastasis has great influence on the prognosis of spinal and pelvic ostesarcoma.Zils et al[8] analyzed the clinical data of 20 patients with spinal osteosarcoma and concluded that the patients had metastasis suggests a worse prognosis.A study of pelvic osteosarcoma based on the Japanese Bone Tumor Registry indicated that metastasis leads to a lower rate of surgery, which leads to a lower survival rate[9].Therefor,the study on prediction of distant metastasis of spinal and pelvic osteosarcoma is necessary. We aim to construct a nomogram to predict the risk of distant metastasis of spinal and pelvic osteosarcoma to fill in the blanks.

The Surveillance, Epidemiology, and End Results(SEER) program database contains a large number of information of cancer patients in the United States.Advantages of utilizing such a database include multiinstitutional data and a large patient pool for greater statistical power[10].To get a large sample size, we collected data of patients with spinal and pelvic osteosarcoma from SEER database to build a nomogram to predict the probability of distant metastasis.Furthermore, we also attempted to get a better understanding of the distant metastasis of spinal and pelvic osteosarcoma and provide help for the formulation of clinical treatment plan.

\section{Materials And Methods}

\section{Data Collection}

Data for this study were extracted from the SEER Research Data,18 Registries,Nov 2020 Sub(2000-2018) using SEER ${ }^{\star}$ Stat(version 8.3.9.2) software.The inclusion criteria were as follows:(1)patients diagnosed as primary osteosarcoma with ICD-0-3 morphology codes 9180/9181/9182/9183/ 9185/9186/9187/9192/9193/9194 from 2000 to 2018;(2) codes of primary site were C412 (vertebral column) and C414 (pelvic bones, sacrum, coccyx, and associated joints);

The exclusion criteria were as follows:(1) patients without information about distant metastasis. (2)patients without pathological diagnosis;(3) patients without information about pathological grade;(4) patients without information about age at diagnosis. The detailed screening flowchart is shown in Figure.1.

SEER database is a free and public database,and no personal identifying information is included in this database.Therefor,informed consent or ethical approval was not required for this study.

\section{Study Variables}


The variables in our study included age at diagnosis,sex,race,median household income inflation adj to 2019,primary site,grade,tumor size,number of primary tumors,surgery and survival months.Age was recorded in the form of measurement data according to variable "Age recode with single ages and $85+$ ". Race classification was white,black,and other race.Median household income was divided into $<50000,50000-69999$ and $\geq 70000$ according to variable "Median household income inflation adj to 2019". The primary site was divided into spine and pelvis according to variable "Primary site-labeled".The grade was divided into high grade (including grades I-II) and low grade (including grades III-IV) according to the variable "Grade(thru 2017)" and "Grade Pathological(2018+)". The tumor size was divided into $\leq 5 \mathrm{~cm}, 5-8 \mathrm{~cm}, \geq 8 \mathrm{~cm}$ and unknown according to the variable "CS tumor size(2004-2015)" and "Tumor Size Summary (2016+)".Number of primary tumor was divided into only one and more according to the variable "Sequence number". Whether surgery had been performed determined according to variables "RX Summ-Surg Prim Site(1998+)".Survival months was recorded in the form of measurement data according to variable "Survival months".

\section{Statistical analysis}

The Kaplan-Meier curve was used to compare differences in survival time between patients with metastasis and non-metastasis.All of the patients were divide into training cohort and validation cohort used random number method(set seed=20211020). The Shapiro-Wilk test was used to verify whether the continuous variables conform to normal distribution. If the measurement data conform to normal distribution, $x \pm s$ is used for description,otherwise $M(Q 25, Q 75)$ is used for description. The difference of variables between two groups were compared using Mann-Whitney $\mathrm{U}$ test and Chi-square test.

Least absolute shrinkage, and selection operator (LASSO) regression analysis was performed to select the meaningful factors,prevent overfitting and solve collinearity problems. The risk factors selected from LASSO regression analysis were further analyzed by the multivariate logistic regression analysis to confirm independent risk factors. The multivariate logistic regression model is used to calculate the odds ratio of each variable with a corresponding $95 \%$ confidence interval(Cl).

Furthermore, we constructed nomogram based on the results obtained from the multivariate logistic regression analysis.

We validated the nomogram in the training and validation cohorts internally and externally.The C-index of nomogram and calibration curves for the training and validation cohorts were used to evaluate the predictive reliability of nomogram. The predictive discrimination of the nomogram was assessed by receiver operating characteristic curve(ROC).

Moreover, we utilized decision curve analysis(DCA) to assess the clinical benefits of the nomogram.

The random number method,the Shapiro-Wilk test,the Mann-Whitney U test,Chi-square test and KaplanMeier curve were performed by SPSS statistics software version 22.0(IBM Corporation,Armonk,New 
York,United State of American), and the LASSO regression analysis,multivariate logistic regression were and construction and validation of the nomogram were performed by $\mathrm{R}$ version 4.1 .1 (http://www.rproject.org/). A two-sided $p$ value $<0.05$ was considered to have statistical significance.

\section{Results}

A total of 358 patients were included in our study. The Kaplan-Meier curve showed that the survival time of non-metastatic patients with spinal and pelvic osteosarcoma was longer than that of metastatic patients $(P<0.001$,Figure 2$)$. All patients $(n=358)$ were divided into training cohort $(n=269)$ and validation cohort $(n=89)$ used random number method.Age was described used M(Q25,Q75) because it did not conform to normal distribution proved by the Shapiro-Wilk test in training cohort and validation cohort.There was no significant differences between the two cohorts in all variables. $(P>0.05$,Table 1$)$.

The LASSO regression selected five meaningful variables including sex,race,grade,number of primary tumor and surgery in the training cohort(Figure 3A,3B).According to the result of multivariate logistic regression analysis,patients with factors including "male","other races","high grade","single primary tumor","no surgery" were more likely to have distant metastasis.Furthermore,"no surgery”(yes, $O R=0.175,95 \% C l=0.095-0.321, p=0.000$ ) was independent risk factors for distant metastasis in patients with spinal and pelvic osteosarcoma(Table 2).

The nomogram assigned a score to each variable to predict the probability of distant metastasis of patients with spinal and pelvic osteosarcoma(C-index=0.752,Figure 4).The C-index of nomogram and calibration curves for the training and validation cohorts showed the good agreement between the predicted results and the actual results(Figure 5A,5B).The area under the receiver operating characteristic curve(AUC) values were $0.748(95 \% \mathrm{Cl}=0.687-0.817)$ and $0.758(95 \% \mathrm{Cl}=0.631-0.868)$ in the training and validation cohorts respectively(Figure 6), which showed the good predictive effect of nomogram. The DCA showed that the nomogram has a clinical usefulness and net benefit when the threshold probability about 0.10 to 0.73 (Figure 7 ). 
Table 1

Baseline characteristics of the training cohort and validation cohort.

\begin{tabular}{|c|c|c|c|c|c|}
\hline Variables & Level & Total(n=358) & training( $n=269)$ & Validation $(n=89)$ & $\begin{array}{l}P \\
\text { value }\end{array}$ \\
\hline $\begin{array}{l}\text { Age } \\
\text { (years,M(Q25,Q75)) }\end{array}$ & & $45(22,63)$ & $45(22,63)$ & $42(20,63)$ & 0.971 \\
\hline \multirow[t]{2}{*}{ Sex } & Male & $179(50.00 \%)$ & $132(49.07 \%)$ & $47(52.81 \%)$ & \multirow[t]{2}{*}{0.541} \\
\hline & Female & $179(50.00 \%)$ & $137(50.93 \%)$ & $42(47.19 \%)$ & \\
\hline \multirow[t]{3}{*}{ Race } & White & $272(75.98 \%)$ & $201(74.72 \%)$ & $71(79.78 \%)$ & \multirow[t]{3}{*}{0.532} \\
\hline & Black & $52(14.53 \%)$ & $40(14.87 \%)$ & $12(13.48 \%)$ & \\
\hline & Other & $34(9.50 \%)$ & $28(10.41 \%)$ & $6(6.74 \%)$ & \\
\hline \multirow{3}{*}{$\begin{array}{l}\text { Median household } \\
\text { income }\end{array}$} & $<50000$ & $53(14.80 \%)$ & $39(14.50 \%)$ & $14(13.73 \%)$ & \multirow[t]{3}{*}{0.792} \\
\hline & $\begin{array}{l}50000- \\
69999\end{array}$ & $160(44.69 \%)$ & $123(45.72 \%)$ & $37(41.57 \%)$ & \\
\hline & $\geq 70000$ & $145(40.50 \%)$ & 107(39.78\%) & $38(42.70 \%)$ & \\
\hline \multirow[t]{2}{*}{ Metastasis } & Yes & $100(27.93 \%)$ & $75(27.88 \%)$ & $25(28.09 \%)$ & \multirow[t]{2}{*}{0.970} \\
\hline & No & $258(72.07 \%)$ & 194(72.12\%) & $64(71.91 \%)$ & \\
\hline \multirow[t]{2}{*}{ Primary site } & Spine & $74(20.67 \%)$ & $56(20.82 \%)$ & $18(20.22 \%)$ & \multirow[t]{2}{*}{0.905} \\
\hline & Pelvic & $284(79.33 \%)$ & 213(81.41\%) & 71(79.78\%) & \\
\hline \multirow[t]{2}{*}{ Grade } & High & $324(90.50 \%)$ & 241(89.59\%) & $83(93.26 \%)$ & \multirow[t]{2}{*}{0.306} \\
\hline & Low & $34(9.50 \%)$ & $28(10.41 \%)$ & $6(6.74 \%)$ & \\
\hline \multirow[t]{4}{*}{ Tumor size(cm) } & $\leq 5$ & $38(10.61 \%)$ & $25(9.29 \%)$ & $13(14.61 \%)$ & \multirow[t]{4}{*}{0.316} \\
\hline & $5-8$ & $49(13.69 \%)$ & $36(13.38 \%)$ & $13(14.61 \%)$ & \\
\hline & $\geq 8$ & $168(46.93 \%)$ & $125(46.47 \%)$ & $43(48.31 \%)$ & \\
\hline & Unknown & $103(28.77 \%)$ & $83(30.86 \%)$ & $20(22.47 \%)$ & \\
\hline \multirow{2}{*}{$\begin{array}{l}\text { Number of primary } \\
\text { tumor }\end{array}$} & Only one & $247(68.99 \%)$ & $187(69.52 \%)$ & $60(67.42 \%)$ & \multirow[t]{2}{*}{0.710} \\
\hline & More & $111(31.01 \%)$ & $82(30.48 \%)$ & $29(32.58 \%)$ & \\
\hline \multirow[t]{2}{*}{ Surgery } & Yes & $213(59.50 \%)$ & $153(56.88 \%)$ & $60(67.42 \%)$ & \multirow[t]{2}{*}{0.079} \\
\hline & No & $145(40.50 \%)$ & $116(43.12 \%)$ & $29(32.58 \%)$ & \\
\hline
\end{tabular}


Table 2

Results of multivariate logistic regression analysis.

\begin{tabular}{|c|c|c|c|}
\hline Variables & & $\mathrm{OR}(95 \% \mathrm{Cl})$ & $P$-value \\
\hline \multirow[t]{2}{*}{ Sex } & Male & reference & \multirow[t]{2}{*}{0.122} \\
\hline & Female & $0.628(0.348-1.133)$ & \\
\hline \multirow[t]{3}{*}{ Race } & White & reference & 0.435 \\
\hline & Black & $0.708(0.297-1.687)$ & \multirow[t]{2}{*}{0.096} \\
\hline & Other & $2.132(0.873-5.207)$ & \\
\hline \multirow[t]{2}{*}{ Grade } & Low & reference & \multirow[t]{2}{*}{0.291} \\
\hline & High & $1.895(0.220-3.757)$ & \\
\hline \multirow[t]{2}{*}{ Number of primary tumor } & Only one & reference & \multirow[t]{2}{*}{0.101} \\
\hline & More & $0.575(0.297-1.113)$ & \\
\hline \multirow[t]{2}{*}{ Surgery } & No & reference & \multirow[t]{2}{*}{0.000} \\
\hline & Yes & $0.175(0.095-0.321)$ & \\
\hline
\end{tabular}

\section{Discussion}

This study constructed a nomogram to predict the probability of distant metastasis of spinal and pelvic osteosarcoma.We proved that this is a reliable and efficient prediction model by internal and external validation.Our study indicated that there are five risk factor of distant metastasis including sex,race,grade,number of primary tumor and surgery,but only surgery is an independent risk factor. This result will provide an important theoretical basis for the treatment of spinal and pelvic osteosarcoma.

Many studies have shown that metastasis is associated with a poor prognosis for osteosarcoma. Wenhao Chen et al[11] indicated that distant metastatic osteosarcoma has a poor prognosis,and Kyle R. Duchman et al[12] indicated metastatic disease at diagnosis conferred the poorest prognosis in patients with high-grade osteosarcoma.Spinal and pelvic osteosarcoma has a worse prognosis compered with osteosarcoma of other sites.Our study confirmed that the survival time of patients with metastatic spinal and pelvic osteosarcoma was lower than that of patients with nonmetastatic spinal and pelvic osteosarcoma used the Kaplan-Meier curve.Therefore,we believe that the prognosis of metastatic spinal and pelvic osteosarcoma is worse than non-metastatic spinal and pelvic osteosarcoma.

Our study showed that the median age of patients with spinal and pelvic osteosarcoma was 45 years and there were fewer adolescents. However, osteosarcoma tends to occur in children and young adults according to previous reports $[13,14]$.Therefore, we believe that patients with spinal and pelvic osteosarcoma have a older age than patients with osteosarcoma in other sites, and some previous 
studies have found similar results $[15,16]$. Our study showed that metastasis occurred in $27.93 \%$ of patients with spinal and pelvic osteosarcoma, and previous literature reported $20 \%$ of patients with osteosarcoma have metastases at the time of diagnosis, especially lung metastases[17].We found that $40.50 \%$ of all patients with spinal and pelvic osteosarcoma had no surgical treatment,and that could be because of the difficulty of the operation in axial bones. The spine and pelvis contain many important vessels, nerves, and organs, which making the tumor difficult to remove completely. There is literature suggesting that it is almost impossible to remove the tumor in whole when the tumor involves the bilateral pedicle and involves the vertebral artery foramen or lamina,or the odontoid process[18].

We used LASSO regression analysis to identify five risk factors for metastasis of spinal and pelvic osteosarcoma:sex,race,grade,number of primary tumor and surgery. Our study showed that female patients and black patients with spinal and pelvic osteosarcoma were less likely to metastasis, which may provide some reference for clinical treatment decisions. Tumor grade is the description of a tumor based on how abnormal the tumor cells and the tumor tissue look under a microscope[4].In our study,the high grade was a risk factor for metastasis of spinal and pelvic osteosarcoma,which should be related to the rapid growth and diffusion of poorly differentiated or undifferentiated tumor cells. In addition,we found that patients with single primary tumors are more likely to metastasize than patients with multiple primary.There are no studies on relationship between number of primary tumor and distant metastasis of spinal and pelvic osteosarcoma,currently. We thought that molecular and biochemical studies of tumors may be needed to explain this.

In our study,surgery was confirmed to be an independent risk factor for distant metastasis of spinal and pelvic osteosarcoma by multivariate logistic regression analysis.Patients who underwent surgery had significantly lower rates of metastasis than those who did not undergo surgery.Therefor,surgery for spinal and pelvic osteosarcoma may be preferred based on this result.The main treatment for the primary osteosarcoma is chemotherapy and surgery[20],of which complete resection of the primary tumor blocks the progression of osteosarcoma including metastases[21, 22].Due to the specificity of spinal and pelvic anatomy, only intrafocal resection can be achieved in early stage.But these surgical methods lead to incomplete tumor resection and easy to postoperative tumor recurrence and distant metastasis[23].With the development of surgical techniques,total en bloc spondylectomy(TES) was invented and considered to be an aggressive surgical technique that may be employed in the treatment of spinal neoplasms[24, 25].Several large sample case studies showed extensive resection of osteosarcoma or at least marginal resection based on TES can be performed to minimize tumor cell contamination from surgical procedures and reduce the local recurrence rate and improve the survival rate of patients[26, 27].Pelvic osteosarcoma surgery mainly includes limb salvage surgery and amputation.A number of long-term clinical studies have shown that limb salvage is similar to amputation[28], and Hindquarter amputation should be considered when limb salvage does not guarantee a satisfactory resection margin[29].

The nomogram is well known for its predictive accuracy and has made remarkable contributions to modern medical[30, 31].Jiang $R$ et al[32] constructed a nomogram for pelvic and spinal ewing sarcoma patients to facilitate advances in individual treatment by quantitatively analyzing survival predictors. Li W 
et al[33] constructed a novel nomogram to predict risk factors for chondrosarcoma patients and guided clinicians to optimize personalized treatment and make superior clinical related decisions. To the best of our knowledge, this is the first study constructing a nomogram to predict the probability of metastasis for

patients with spinal and pelvic osteosarcoma, and personalized treatment can be developed based on this predictive model.Undoubtedly, compared with general treatment, personalized treatment is more rational and specific[34].For example,we should pay more attention to check for metastasis when patients who had not undergone surgery were reviewed.In addition,we need to be more targeted in our screening and treatment plans for high-grade spinal and pelvic osteosarcoma patients. Therefor,this predictive model is of great significance to orthopedic surgeons in the clinical management of spinal and pelvic osteosarcoma.

We have validated the reliability and validity of nomogram both internally and externally.C-index can evaluate the probability that the predicted results are consistent with the actual observed results,while assessing calibration is an important component of deriving and validating clinical prediction models[35].C-index of nomogram in our study is greater than 0.7 and the calibration curves of nomogram in training cohort and validation cohort showed a high degree of agreement between the predicted and actual observed metastasis rates of the training and validation cohorts, indicating that the nomograme are reliable.Receiver operating characteristic (ROC) curve is ofen used to discriminate performance of clinical prediction models[36].In our study,AUC values were greater than 0.7 in the training and validation cohorts, which indicated a good predictive effect.Decision curve analysis was developed as a method to determine whether use of a prediction model in the clinic to inform clinical decision-making would do more good than harm[37].The DCA showed that the nomogram has a clinical usefulness and net benefit in most cases. To sum up,the nomogram constructed in our study has good reliability, validity and clinical benefit.

However, there are some limitations to our study.First of all,The sample size of our study was not as large as that of other studies based on SERR databases because patients with spinal and pelvic osteosarcoma are extremely rare.However,our study has been the largest sample size study on distant metastasis of spinal and pelvic osteosarcoma.Secondly, some patients lacking pathological grade were excluded from the study, and some patients included in our study lacked tumor size data,which may lead to inaccurate inferences.Thirdly,the SEER database(18Registries, Nov 2020 Sub(2000-2018)) lacks other important information, such as data about neurological function;specific surgical methods;pivotal serological biomarkers;radiation or chemotherapy;local recurrence,which leaded to some relevant bias.Finally,because of both training and validation cohorts are from the same database, it is necessary to get data from another database for validation.

\section{Conclusion}

Surgery is the independent risk factor of distant metastasis of spinal and pelvic osteosarcoma.A nomogram to predict distant metastasis in patients with spinal and pelvic osteosarcoma was constructed and validated based on risk factors, including sex,race,grade,number of primary tumor and 
surgery based on data obtained from the SEER database. Through internal and external verification,we think this nomogram is a reliable and useful tool for orthopedic surgeons that can improve prediction of the probability of distant metastasis in patients with spinal and pelvic osteosarcoma.

\section{Abbreviations}

SEER database:Surveillance, Epidemiology, and End Results database

LASSO regression:Least absolute shrinkage and selection operator regression

ROC curve:Receiver operating characteristic curve

DCA:Decision curve analysis

AUC:Area under the receiver operating characteristic curve

TES:Total en bloc spondylectomy

\section{Declarations}

\section{Ethics approval and consent to participate}

SEER database is a free and public database,and no personal identifying information is included in this database.Therefor,informed consent or ethical approval was not required for this study.

\section{Consent for publication}

Not applicable

\section{Availability of data and materials}

The datasets analysed during the current study are available in the Surveillance, Epidemiology, and End Results(SEER) database,[https://

seer.cancer.gov/].

\section{Competing interests}

There is no competing interests between this article and other individuals or organizations.

\section{Funding}

Not applicable

Authors' contributions 
Yu Lin:Study creativity and design,Data collection and analysis,Manuscript writing.

Wenge Liu:Study creativity and design,Manuscript review.

Zhenyu Wang:Study creativity and design,Data collection.

Gang Chen:Data collection and analysis.

\section{Acknowledgments}

We thank the SEER database for making information available.We are grateful to all people who contributed to this article.

\section{References}

1. ESMO/European Sarcoma Network Working Group.Bone sarcomas:ESMO Clinical Practice Guidelines for diagnosis,treatment and follow up.Ann Oncol.2014;25(Supplement 3):iii113-123.

2. Joseph Schwab,Alessandro Gasbarrini,Stefano Bandiera,Luca Boriani, Luca Amendola,Piero Picci,et al.Osteosarcoma of the mobile spine. Spine.2012;37(6):E381-E386.

3. MC Parry,M Laitinen,J Albergo,L Jeys,S Carter,CL Gaston,et al.Osteosarcoma of the pelvis.Bone Joint J.2016;98(B):555-63.

4. Lu S,Wang Y,Liu G,Wang L,Wu P,Li Y,et al.Construction and Validation of Nomogram to Predict Distant Metastasis in Osteosarcoma: A Retrospective Study.J Prthopaed Surg Res.2021;16(1):231.

5. Zhang C,Guo X,Xu Y,Han X,Cai J,Wang X,Wang G.Lung metastases at the initial diagnosis of highgrade osteo-sarcoma:Prevalence,risk factors and prognostic factors.A large population-based cohort study.Sao Paulo Med J.2019;137(5):423-429.

6. Jiang J,Pan H,Li M,Qian B,Lin X, Fan S.Predictive model for the 5-year survival status of osteosarcoma patients based on the SEER database and XGBoost algorithm. Scientific Reports.2021;11:5542.

7. Yang QK,Lai QY,Wang Y,Wang Y,Yao ZX,Zhang XJ.Establishment and validation of prognostic nomograms to predict overall survival and cancer-specific survival for patients with osteosarcoma.NEOPLASMA. 2021;;68(2):434-446.

8. Zils K,Bielack S,Wilhelm M,Werner M,Schwarz R,Windhager R,et al.Osteosarcoma of the mobile spine.Ann Oncol.2013;24(8):2190-2195.

9. Satoshi Takenaka,Hironari Tamiya,wwToru Wakamatsu,Sho Nakai,Yoshinori Imura,Hidetatsu Outani,et al.Impact of Surgical Resection and Reasons for Poor Prognosis of Pelvic Osteosarcoma Based on the Bone Tumor Registry in Japan.Cancers.2021;13:3320.

10. Runyi Jiang,Shaohui He,Haitao Sun,Haiyi Gong,Xinghai Yang,Xiaopan Cai,et al.Identifying the Risk Factors and Estimating the Prognosis in Patients with Pelvis and Spine Ewing Sarcoma.Spine.2021;46:1316. 
11. Wenhao Chen,Yuxiang Lin.Nomograms predicting overall survival and cancer-specific survival in osteosarcoma patients (STROBE).Medicine. 2019;;98:e16141.

12. Duchman $\mathrm{K}, \mathrm{Gao}$ Y,Miller B.Prognostic factors for survival in patients with high-grade osteosarcoma using the surveillance, epidemiology, and end results (SEER) program database.Cancer Epidemiology.2015;39(4): -599.

13. Jafari F,Javdansirat S,Sanaie S,Naseri A,Shamekh A,Rostamzadeh D,et al.Osteosarcoma:A comprehensive review of management and treatment strategies.Annals of Diagnostic Pathology.2020;49:151654.

14. Mirabello L,Troisi RJ,Savage SA.Osteosarcoma incidence and survival rates from 1973 to 2004: data from the Surveillance, Epidemiology, and End Results Program.Cancer-Am Cancer Soc.2009;115:1531-1543.

15. Dekutoski MB,Clarke MJ,Rose P,Luzzati A,Rhines LD,Varga PP,et al.Osteosarcoma of the spine:prognostic variables for local recurrence and overall survival,a multicenter ambispective study.J Neurosurg Spine.2016;25(1):59-68.

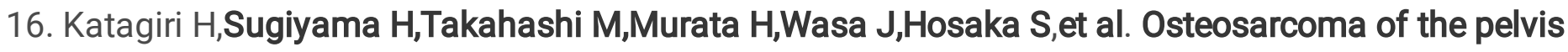
treated successfully with repetitive intra-arterial chemotherapy and radiation therapy:A report of a case with a 21-year follow-up.J Orthop Sci.2015;20:568-573.

17. Tian H,Guan D,Li J.Identifying osteosarcoma metastasis associated genes by weighted gene coexpression network analysis(WGCNA).Medicine. 2018.;97(24):e10781.

18. Katonis P,Datsis G,Karantanas A,Kampouroglou A,Lianoudakis S, Licoudis S, et al.Spinal osteosarcoma.Clin Med Insights Oncol.2013;7: -208.

19. Lindsey BA,JE Markel,ES Kleinerman.Osteosarcoma overview. Rheumatology and therapy.2017;4(1):25-43.

20. Marulanda GA,Henderson ER,Johnson DA,Letson GD,Cheong D.Orthopedic surgery options for the treatment of primary osteosarcoma.Cancer Control.2008;15(1):13-20.

21. Spinelli MS,Ziranu A,Piccioli A,Maccauro G.Surgical treatment of acetabular metastasis. Eur Rev Med Pharmacol Sci.2016;20(14):3005-10.

22. Ozaki T,Flege S,Liljenqvist U,Hillmann A,Delling G,Salzer-Kuntschik M,et al.Osteosarcoma of the spine:experience of the Cooperative Osteosarcoma Study Group.Cancer.2002,94(4):1069-1077.

23. Luzzati AD,Shah S,Gagliano F,Perrucchini G,Scotto G,Alloisio M.Multilevel en bloc spondylectomy for tumors of the thoracic and lumbar spine is challenging but rewarding.Clin Orthop Relat Res.2015;473: $-\mathbf{8 6 7 .}$

24. Yin H,Yang $X$,Xu W,Li B,Li B,Wang T,et al.Treatment and outcome of primary aggressive giant cell tumor in the spine.Eur Spine J. 2015.;24:1747-1753.

25. Lim JB,Sharma H,MacDuff E,Reece AT.Primary osteosarcoma of the spine:a review of 10 cases.Acta Orthop Belg.2013;79(4):457-462. 
26. Feng D,Yang X,Liu T,Xiao J,Wu Z,Huang Q,et al.Osteosarcoma of the spine:surgical treatment and outcomes.World J Surg Oncol.2013;11(1):89.

27. Rougraff BT,Simon MA,KneisI JS,Greenberg DB,Mankin HJ.Limb salvage compared with amputation for osteosarcoma of the distal end of the femur. A long-term oncological,functional,and quality-of-life study.J Bone Joint SurgAm.1994;76(5):649-656.

28. Sherman $\mathrm{CE}, \mathrm{O}^{\prime}$ Connor $\mathrm{MI}$,Sim $\mathrm{FH}$.Survival,local recurrence,and function after pelvic limb salvage at 23 to $\mathbf{3 8}$ years of followup. Clin Orthop Relat Res.2012;470(3):712-727.

29. Li G,Tian ML,Bing YT,Wang HY,Yuan CH,Xiu DR.Nomograms predict survival outcomes for distant metastatic pancreatic neuroendocrine tumor: A population based STROBE compliant study.Medicine.2020;99(13):e19593.

30. Serenari M,Han KH,Ravaioli F,Kim SU,Cucchetti A,Han DH,et al.A novel nomogram based on liver stiffness to predict the comprehensive complication index after liver resection in patients with hepatocellular carcinoma.Dig Liver Dis.2020;52(1):e17.

31. Jiang R,He S,Sun $H$,Gong $H$,Yang $X$,Cai $X$,et al. Identifying the Risk Factors and Estimating the Prognosis in Patients with Pelvis and Spine Ewing Sarcoma.spine.2021;46(19):1315-1325.

32. Li W,Dong S,Wang $H, W u R$,Wu $H$,Tang ZR,et al.Risk analysis of pulmonary metastasis of chondrosarcoma by establishing and validating a new clinical prediction model: a clinical study based on SEER database.BMC Musculoskeletal Disorders.2021;22:529.

33. Thewes B,Husson O,PoortH,Custers JAE,Butow PN,McLachlan SA,et al.Fear of Cancer Recurrence in an Era of Personalized Medicine.JOURNAL OF CLINICAL ONCOLOGY.2017;35:3275-3278.

34. Austin PC,Harrell FE Jr,van Klaveren D.Graphical calibration curves and the integrated calibration index (ICI) for survival models. Stat Med.2020;39(21):2714-2742.

35. Cook NR.Use and misuse of the receiver operating characteristic curve in risk prediction.Circulation.2007;115(7):928-35.

36. Vickers AJ,Elkin EB.Decision curve analysis:a novel method for evaluating prediction models.Med Decis Making.2006;26(6):565-74.

\section{Figures}




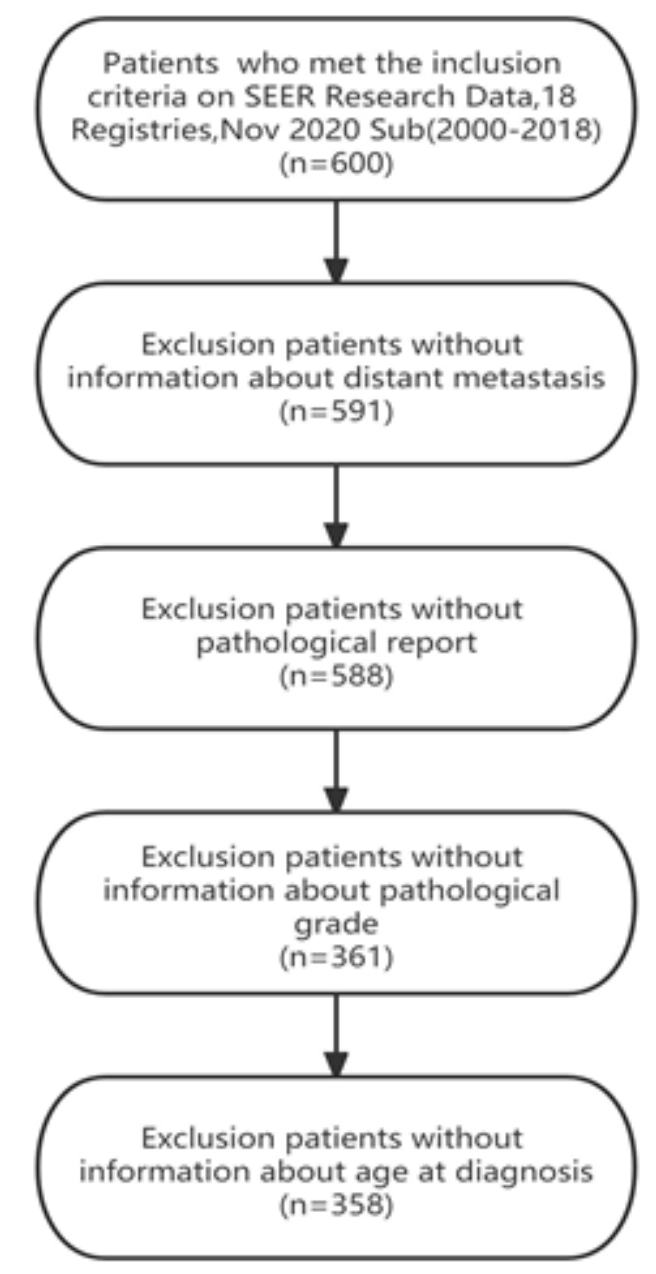

Figure 1

Flow diagram of patient selection 


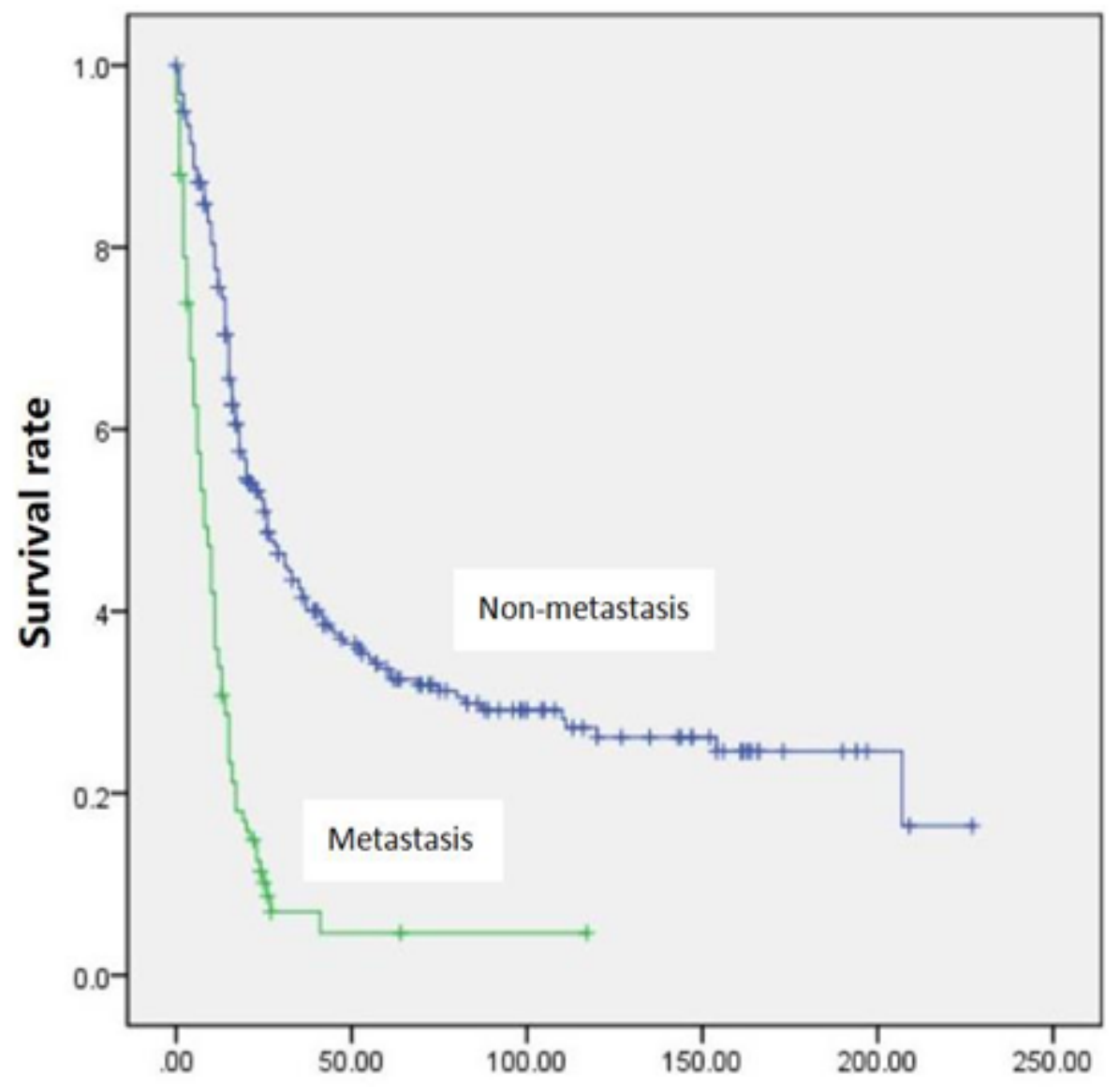

Survival months

Figure 2

Kaplan-Meier curve of non-metastatic and metastatic patients with spinal and pelvic osteosarcoma.
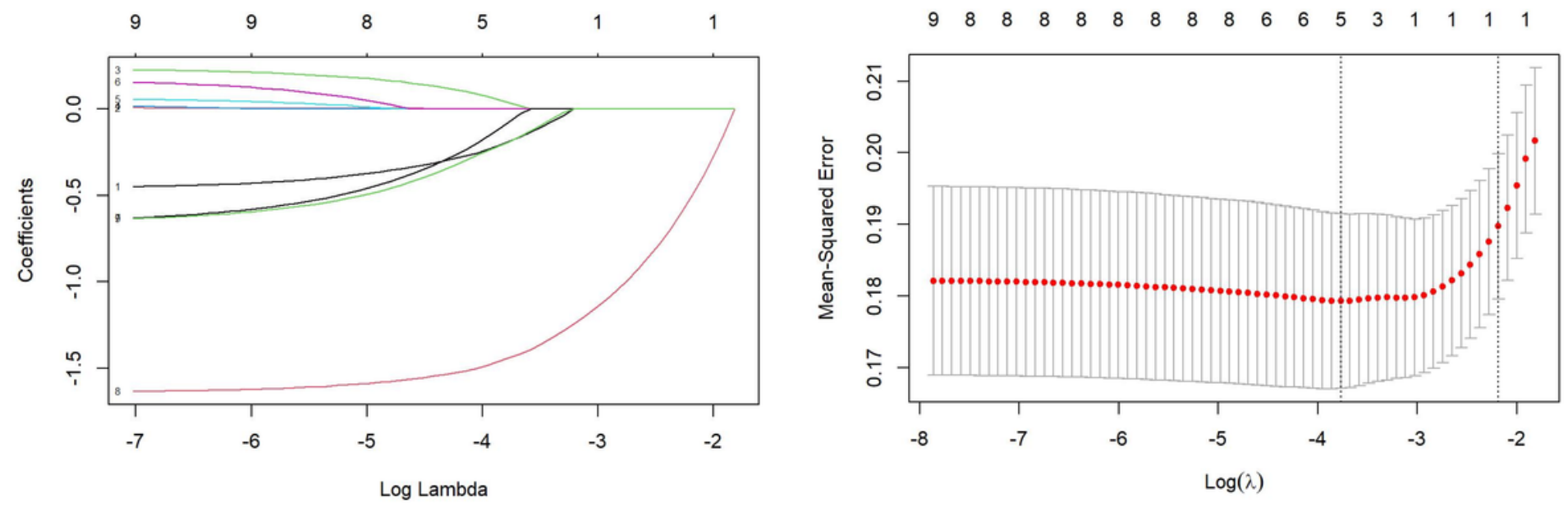

Figure 3

The results of LASSO regression. 


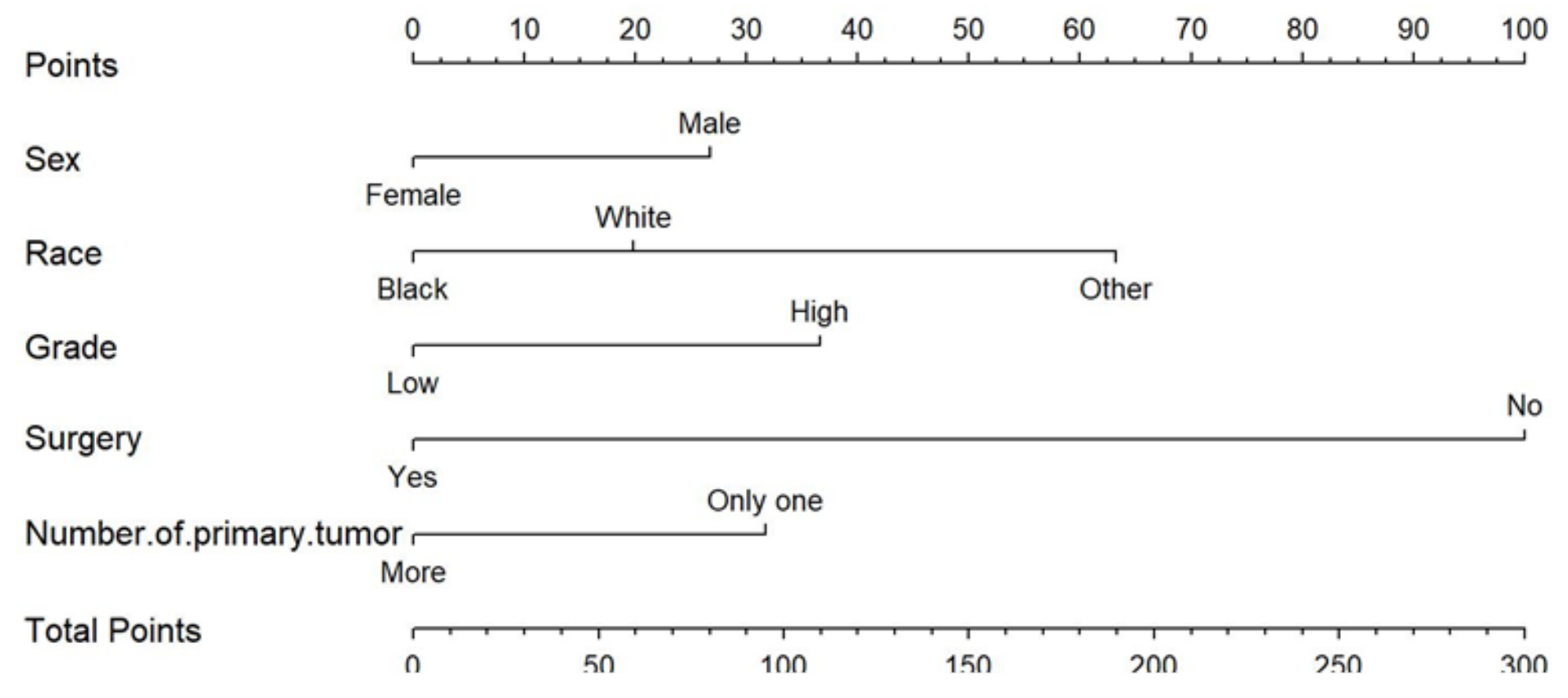

\section{Figure 4}

The nomogram for predicting the probability of metastasis. 


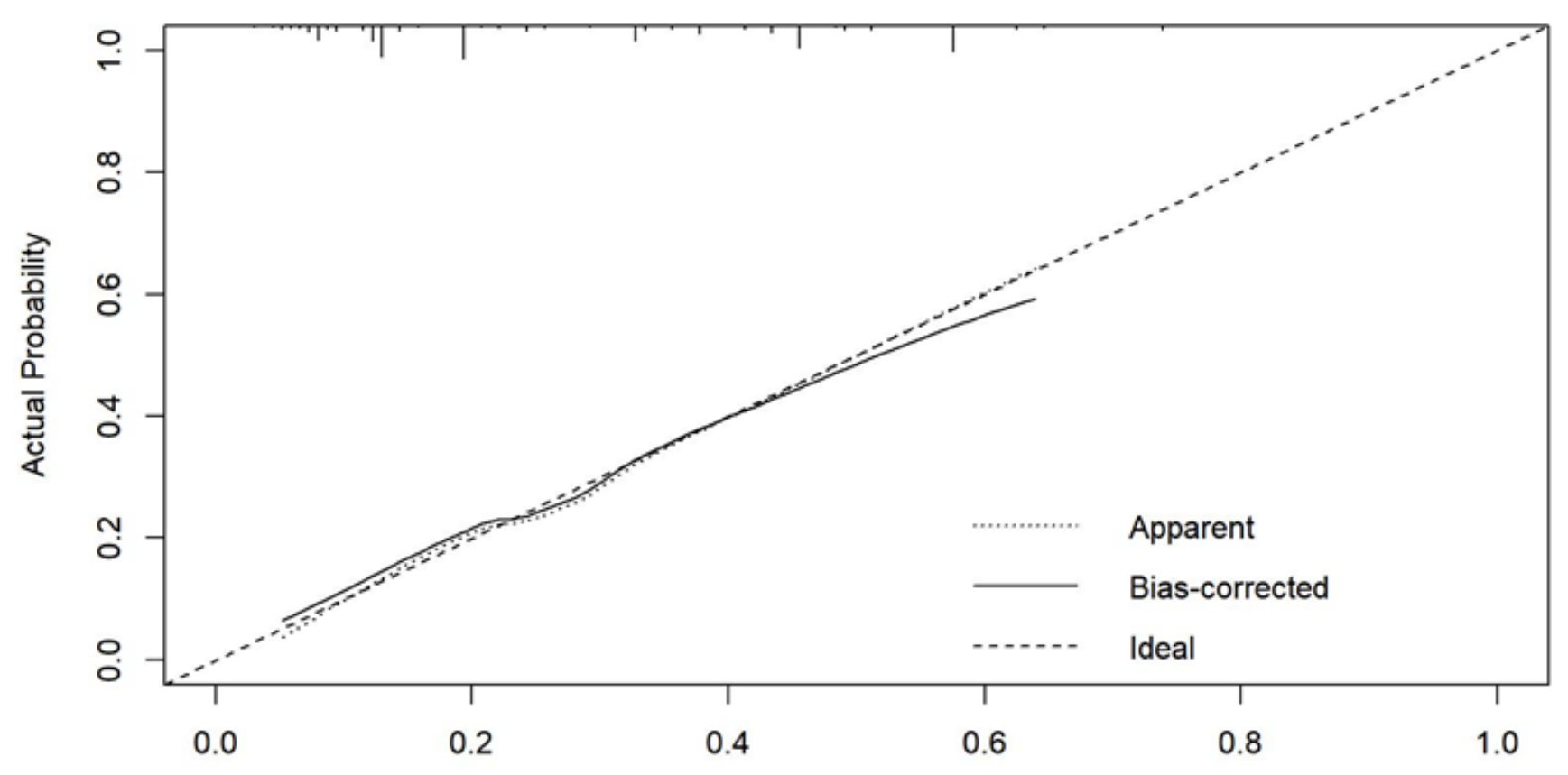

Predicted Probability

$B=1000$ repetitions, boot

Mean absolute error $=0.014 n=269$

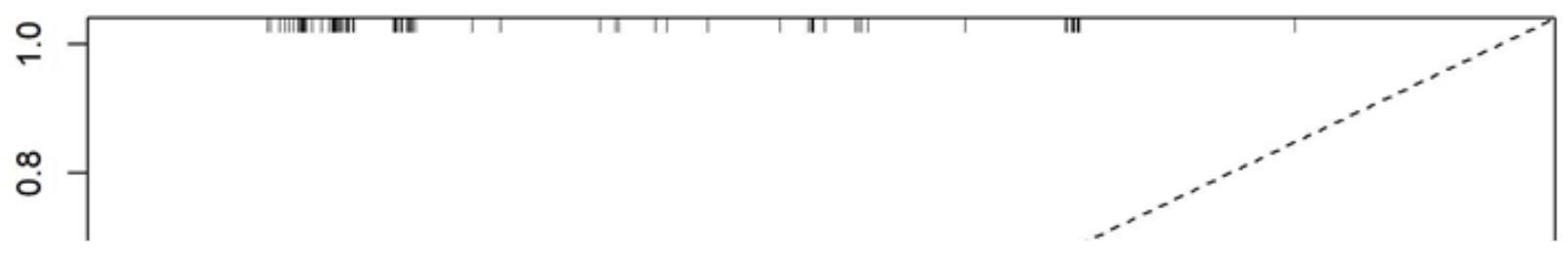

\section{Figure 5}

A.The calibration curves of nomogram for training cohort.

B.The calibration curves of nomogram for validation cohort. 


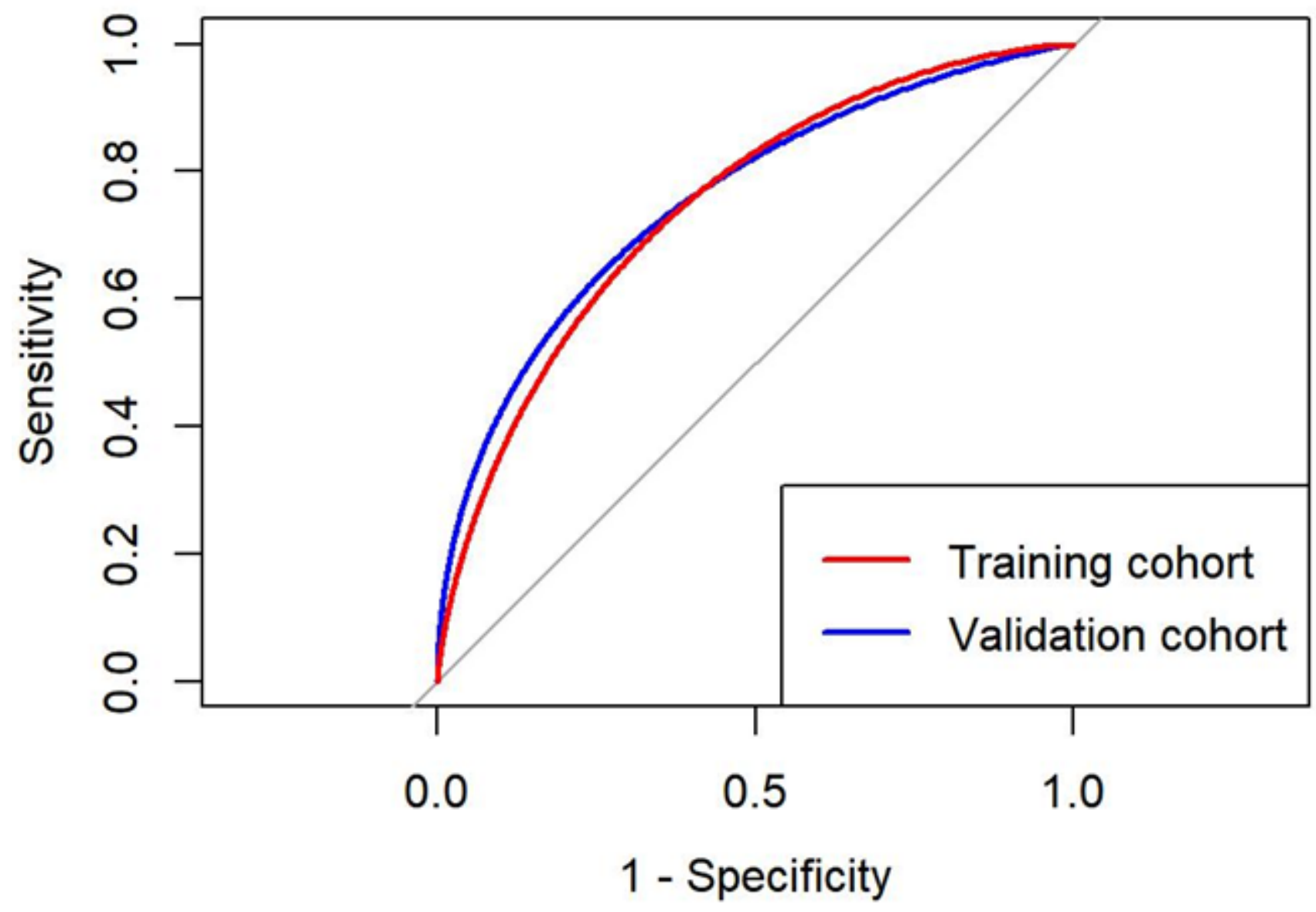

Figure 6

ROC of nomogram. 


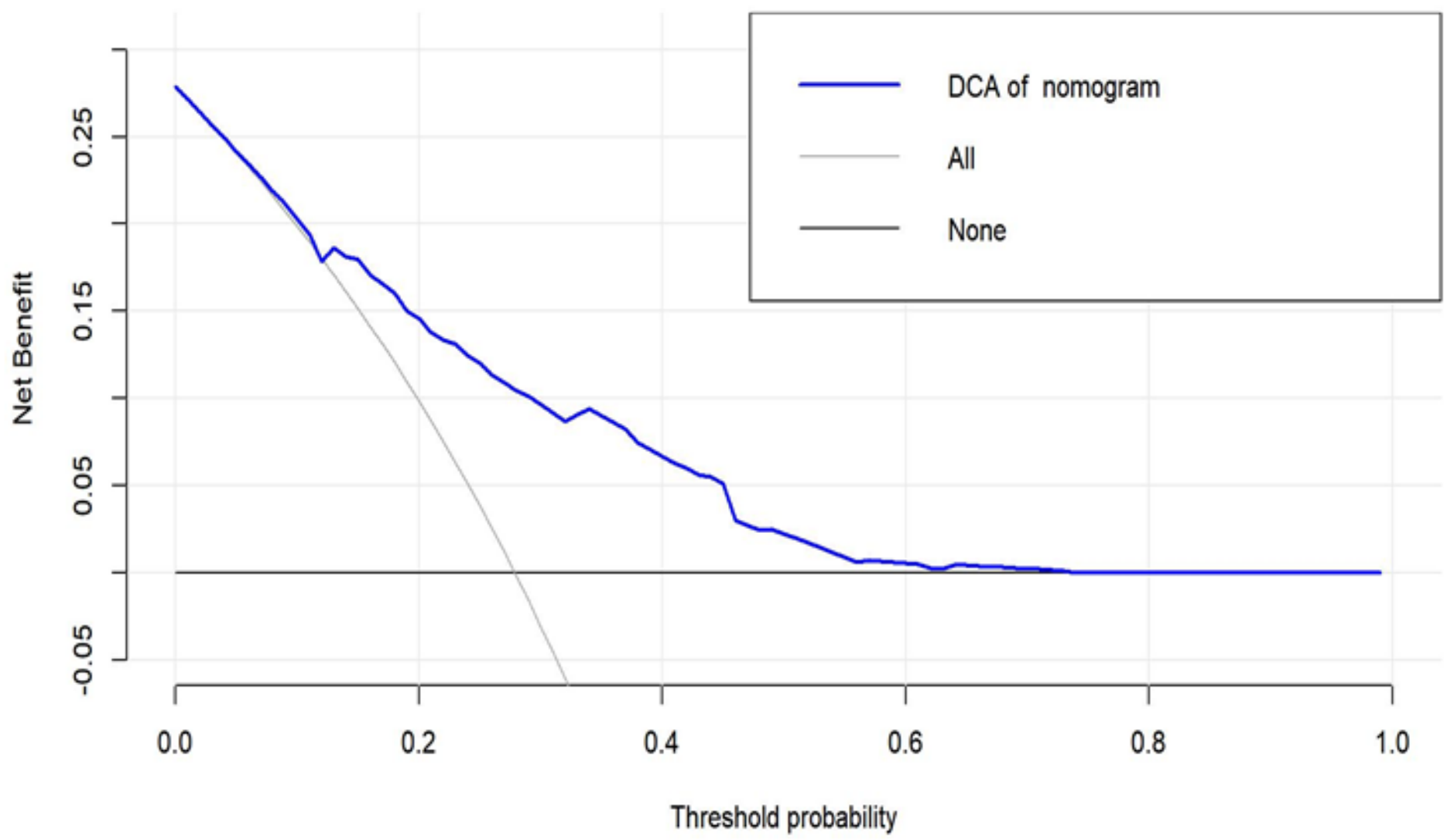

Figure 7

DCA of nomogram in training cohort. 